\title{
Concepção interdisciplinar da interface do sistema de gestão da informação
}

\author{
Ivette Kafure
}

Resumo: Este trabalho ilustra uma pesquisa interdisciplinar sobre os usuários e as interfaces de sistemas de gestão da informação, levando em consideração a ergonomia cognitiva, o design emocional, o processo de desenvolvimento e avaliação das interfaces de sistemas da informação, o custo, a praticidade, a disponibilidade, a acessibilidade e a usabilidade da informação. A proposta interdisciplinar incorpora contribuições da Ciência da Informação, Artes, Comunicação, Psicologia Cognitiva, Educação, Computação e áreas afins. Para alcançar este objetivo têm sido realizadas diversas experiências nas quais têm sido considerados certos atributos subjacentes à atuação do usuário, tais como, motivação, raciocínio, atenção e memorização. Muitas vezes um sistema de informação é conhecido pelos usuários quando este já está em funcionamento, isto que pode gerar a existência de uma discrepância entre as necessidades e expectativas dos usuários e as dificuldades encontradas para acessar à informação. Esta pesquisa avalia o processo de desenvolvimento de interfaces de sistemas de gestão da informação desde o início, integrando o usuário e sua maneira de entender e executar a tarefa, com a utilização e aplicação de técnicas tais como, a prototipagem em papel, o grupo focal, a entrevista, o arranjo de cartões, a observação sistemática, a análise da tarefa e da atividade. Isto gerou recomendações fundamentadas na junção da lógica dos usuários, dos projetistas e dos profissionais da informação, sendo revisada a literatura sobre a área, as publicações que agrupam recomendações, e analisados os resultados de avaliação da usabilidade em diferentes experiências. A aplicação dessas recomendações têm agregado valor à compreensão e análise dos aspectos envolvidos na concepção de interfaces de sistemas de gestão da informação e ao aumento no grau de satisfação dos usuários.

Palavras-chave: Gestão da Informação. Estudo de Usuários. Interação Humano-Computador. Design Emocional. Usabilidade.

Abstract: This research shows that, there is a conceptual mismatch between the way the user thinks and the way the user interface of the information system was designed. Having this situation, this investigation emphasizes the importance of considering emotional design and the underlying attributes of the user, such as, memorization, reason, attention and motivation, since these can deeply interfere with in the human-computer interaction. It is also illustrated, how were applied diverse techniques, in different stages of various experiences to develop interface projects, such as, paper prototyping, focal group, interview, card sorting, observation, task and activity analysis to bridge the gap between the user needs and the user satisfaction. This proposal incorporates contributions of the Science of the Information, Arts, Communication, Cognitive Psychology, Education, Computation and related areas. This generated recommendations based on the junction of the logic of the users, the designers and the professionals of the information. The application of these recommendations has added value to the understanding and analysis of the involved aspects in the conception of interfaces of information management systems and increases the user satisfaction.

Keywords: Information Management Systems. User Studies. Human-Computer Interaction. Emotional Design. Usability.

Resumen: Este trabajo muestra una investigación interdisciplinaria sobre los usuarios y las interfaces de sistemas de gestión de la información, llevando en consideración la ergonomía cognitiva, el diseño emocional, el proceso de desarrollo y evaluación de interfaces de sistemas de información, el costo, la practicidad, la disponibilidad, la accesibilidad y la usabilidad de la información. Esta propuesta interdisciplinar incorpora contribuciones de la Ciencia de la Información, Artes, Comunicación, Psicología Cognitiva, Educación, Computación y áreas afines. Para alcanzar este objetivo han sido realizadas diversas experiencias en las que han sido considerados ciertos atributos subyacentes del usuario, tales como, motivación, raciocinio, atención y memorización. Muchas veces un sistema de información es conocido por los usuarios cuando este ya está en funcionamiento, esto puede generar la existencia de una discrepancia entre las necesidades y expectativas de los usuarios y las dificultades encontradas en el acceso a la información. Esta investigación evalúa el proceso de desarrollo de las interfaces de sistemas de gestión de la información desde el inicio, integrando el usuario y su manera de ejecutar la tarea, con la utilización de técnicas, tales como, el prototipo en papel, grupo focal, 
entrevista, ordenación de tarjetas, observación sistemática, análisis de la tarea y la actividad. Esto ha generado recomendaciones fundamentas en la lógica de los usuarios, los analistas y los profesionales de la información. La aplicación de estas recomendaciones ha enriquecido el proceso creativo de las interfaces de sistemas de gestión de la información y ha aumentado el grado de satisfacción de por parte de los usuarios.

Palavras Clave: Gestión de la Información. Estudio de Usuarios. Interacción Humano-computador. Diseño Emocional. Usabilidad.

\section{Introdução}

Muitas vezes, um sistema de informação é conhecido pelo usuário quando já está em funcionamento. Isto pode gerar uma discrepância entre as necessidades e expectativas do usuário e as dificuldades encontradas por ele para acessar a informação (Norman, 1986; Kafure, 2000). Esta pesquisa iniciou-se em 1990 quando, trabalhando como analista de sistemas, constatei que o desenvolvimento de sistemas de informação fazia-se sem estudo prévio do perfil do usuário, mas com o foco nas funcionalidades do sistema, as quais pareceriam fáceis de desenvolver com o auxilio dos computadores, no menor tempo possível. Os resultados deste procedimento causavam ansiedade no usuário, para quem era difícil o entendimento dos aplicativos, e alto custo de treinamento, pois várias semanas eram necessárias para o usuário estar apto a cumprir com as tarefas exigidas. Finalmente, se o software não satisfazia os níveis de produção da instituição, ela, muitas vezes, comprava um software desenvolvido por terceiros ou por outras culturas e/ou países supostamente mais avançados na área, de modo a baratear o sistema de informação e cumprir com os requerimentos da instituição.

Para obter uma fundamentação teórico-metodológica sobre como o usuário se comunica, visualiza e entende a funcionalidade de um sistema por meio da interface, foram estudados os princípios de: modelagem do usuário (modelo do Processador Humano - Card, 1983), modelagem da interação homem-computador (Teoria da Ação - Norman, 1983), modelagem da interface (modelo lingüístico - Green, 1995), modelo do processo de concepção do sistema (CLG - Moran, 1981), modelagem de desempenho (GOMS - Card, 1983), guia ergonômico (Scapin 1987). No entanto, essas contribuições estudadas em 1998 careciam principalmente de uma formalização que respondesse ao problema concreto da 
integração explícita dos aspectos do usuário e de sua tarefa no processo de concepção e desenvolvimento de interfaces.

Dentro da perspectiva da ergonomia cognitiva, partindo das contribuições e conceitos introduzidos pelos diferentes trabalhos já realizados, concerne a representação e o estabelecimento de uma correspondência formal de modelos cognitivos (tarefa, usuário) e modelos da interação (conceitualização da interface) incorporando resultados da ergonomia (critérios, recomendações, entre outros), de maneira a permitir a concepção, especificação e desenvolvimento de interfaces que respondam aos objetivos e características do usuário. Neste sentido, podem ser citados os trabalhos de Johnson (Johnson et al., 1988; Johnson e Johnson, 1991), Markopoulos (Markopoulos et al., 1992; Markopoulos e Gikas, 1997), Shneiderman (1998), Scapin e Pierret-Golbreich (1989), que enfatizam a necessidade de/ou propõem representações adequadas da tarefa que permitam a sua manipulação e análise, e de mecanismos que, a partir da representação da tarefa, permitem estabelecer uma especificação conceitual da interface.

$\mathrm{Na}$ continuação desta pesquisa, vêm sendo realizados estudos sobre a inter-relação da Ciência da Informação com disciplinas como a Tecnologia da Informação, as Artes Visuais e a Ergonomia Cognitiva e áreas afins desde 2004, com o objetivo de aumentar a usabilidade do aspecto comunicacional da interface dos sistemas de gestão da informação. O domínio de estudo abordado neste trabalho se propõe a avaliar o processo de desenvolvimento de interfaces de sistemas de gestão da informação que, desde o seu início, integrem o usuário e sua maneira de entender e executar a tarefa (Quadro 1). Para essa integração acontecer, podem ser aplicadas técnicas tais como: a prototipagem em papel, o grupo focal, a entrevista, a análise da tarefa e da atividade (Kafure et al., 2007).

\begin{tabular}{|c|c|}
\hline \multicolumn{2}{|r|}{ Modelo Mental } \\
\hline Necessidades & - acesso à informação \\
\hline Expectativas & $\begin{array}{l}\text { - localizar rapidamente a informação procurada; } \\
\text { - aprendizagem fácil da interface para a gestão da } \\
\text { informação; } \\
\text { - uso fácil da interface para a gestão da informação; } \\
\text { - satisfação na interação humano-computador. }\end{array}$ \\
\hline
\end{tabular}

Quadro 1 - Modelo mental

Fonte: Adaptado de Kafure, 2004, p. 4. 
É preciso começar por entender como os usuários se comportam em relação à informação, e, nessa etapa, eles são a principal fonte de informação. De acordo com Miller (1992) e Cunha (2003), 85\% da informação útil é verbal e informal, antes que as fontes bibliográficas sejam consultadas, daí a importância do contato direto com as pessoas que vão interagir com o sistema de informação.

Um princípio fundamental da ergonomia é conhecer os usuários e o trabalho a ser realizado, que deve ser examinado de acordo com dois componentes básicos: a tarefa e a atividade (Sebillote, 1991, 1995; Guérin et al., 2001). A tarefa faz referência ao que tem que ser feito, ao objetivo a ser alcançado. A atividade é a realização da tarefa.

$\mathrm{Na}$ aplicação da técnica da análise da tarefa e da atividade, tomando como base a metodologia do estudo sobre Usabilidade da imagem na recuperação da informação no catálogo público de acesso em linha (Kafure, 2004), é preciso definir três momentos:

1) Antes de realizar a tarefa: o pesquisador solicita aos usuários que descrevam os passos para realizar a tarefa, com o objetivo de coletar os dados para obter o modelo mental do usuário (Quadro 1).

2) Durante a realização da tarefa: para especificar como os usuários realizam a tarefa (atividade), o pesquisador recorre à observação direta do comportamento enquanto eles executam a tarefa e à entrevista aberta para permitir que se expressem espontaneamente e coloquem seus pontos de vista.

3) Após a realização da tarefa (atividade): o pesquisador questiona os usuários sobre a experiência de realizar a tarefa para avaliar se eles conseguiram realizá-la ou não, e, se for o caso, identificar as dificuldades que eles enfrentaram.

Finalmente, são identificadas as diferenças entre a tarefa e a atividade e como esse conhecimento pode ser útil para aumentar o grau de satisfação do usuário na utilização das interfaces para a gestão da informação.

\section{A tarefa}

As maneiras como o usuário planeja realizar a tarefa têm sido exploradas por meio das seguintes técnicas: pesquisa documental, observação, prototipagem em papel, questionário, entrevista e arranjo de cartões. A informação coletada é submetida à análise de protocolos para se identificar a estrutura da tarefa a partir do ponto de vista dos 
processos que intervêm na sua realização.

O conhecimento que uma pessoa tem acerca do que deve ser feito pode ser estruturado hierarquicamente de acordo com o paradigma do planejamento hierárquico de Sacerdoti (1974). Em outras palavras, a tarefa pode ser descrita em vários níveis de abstração, desde a tarefa-objetivo (nível mais alto), passando pelas subtarefas (nível intermediário), até as mais simples, que podem ser descritas pelas ações elementares simples (nível inferior). Essa decomposição estrutural em vários níveis representa o plano idealizado pelo usuário para realizar o trabalho (Figura 1).

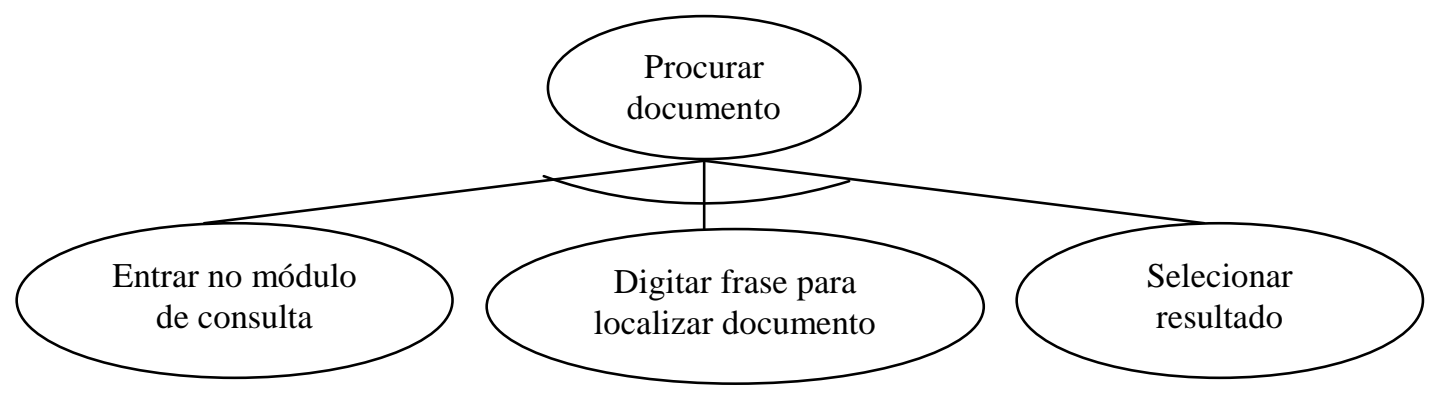

Figura 1 - Análise da tarefa "Procurar documento"

No contexto da concepção da interface, a análise da tarefa pode ser vista como análoga ao desenvolvimento de uma base de conhecimento no contexto da Inteligência Artificial: a aquisição e/ou extração do conhecimento dos usuários (experts) sobre uma tarefa, a representação formal desse conhecimento (base de conhecimento) e a análise e/ou verificação da representação (controle).

Os projetistas ou profissionais da informação devem analisar a tarefa do ponto de vista dos usuários, pois essa análise pode revelar como alcançar os objetivos que os usuários determinaram. Apesar da importância da realização da análise da tarefa no projeto da interface do sistema para a gestão da informação, isto não é muito freqüente (Kafure, 2009a).

\section{A atividade}


Representações mentais são estruturas cognitivas transitórias, constituídas pelo conjunto das informações consideradas pelo sistema cognitivo durante a atividade. Richard (1990) explica que a ação (ou atividade) pode ser vista pela execução da ação, isto é, o modo de realização e o resultado da ação, em outras palavras, o estado a alcançar.

Para se coletar dados sobre a atividade, têm sido utilizadas técnicas como a observação e a descrição em voz alta dos passos que os usuários efetuam na realização da tarefa. Na Figura 2, os resultados da atividade estão esboçados graficamente da mesma maneira que a tarefa, mas com o destaque em negrito e linha dupla para os passos adicionais. Na Figura 3, confrontam-se as possíveis discrepâncias entre a tarefa e a atividade.

Para identificar as possíveis discrepâncias, pode-se aplicar a avaliação comparativa (Kafure, 2004). Disparidades perceptivas existentes entre os usuários das interfaces ocorrem porque a percepção não é uma assimilação mecânica dos dados retinianos, mas a criação de uma imagem estruturada. Perceber consiste em descobrir um padrão estrutural que se ajusta à configuração das formas e cores transmitidas pela retina. Quando a configuração é simples e bem definida, não há lugar para a diversidade, e nem mesmo um esforço intencional permitirá que uma pessoa veja o desenho de um quadrado como outra coisa além de um quadrado (Arnheim, 1989). Esta análise tem permitido orientar a geração e aplicação de recomendações, obtendo-se como resultado a diminuição da distância existente entre o modelo mental do usuário e a imagem da interface (Figura 3).

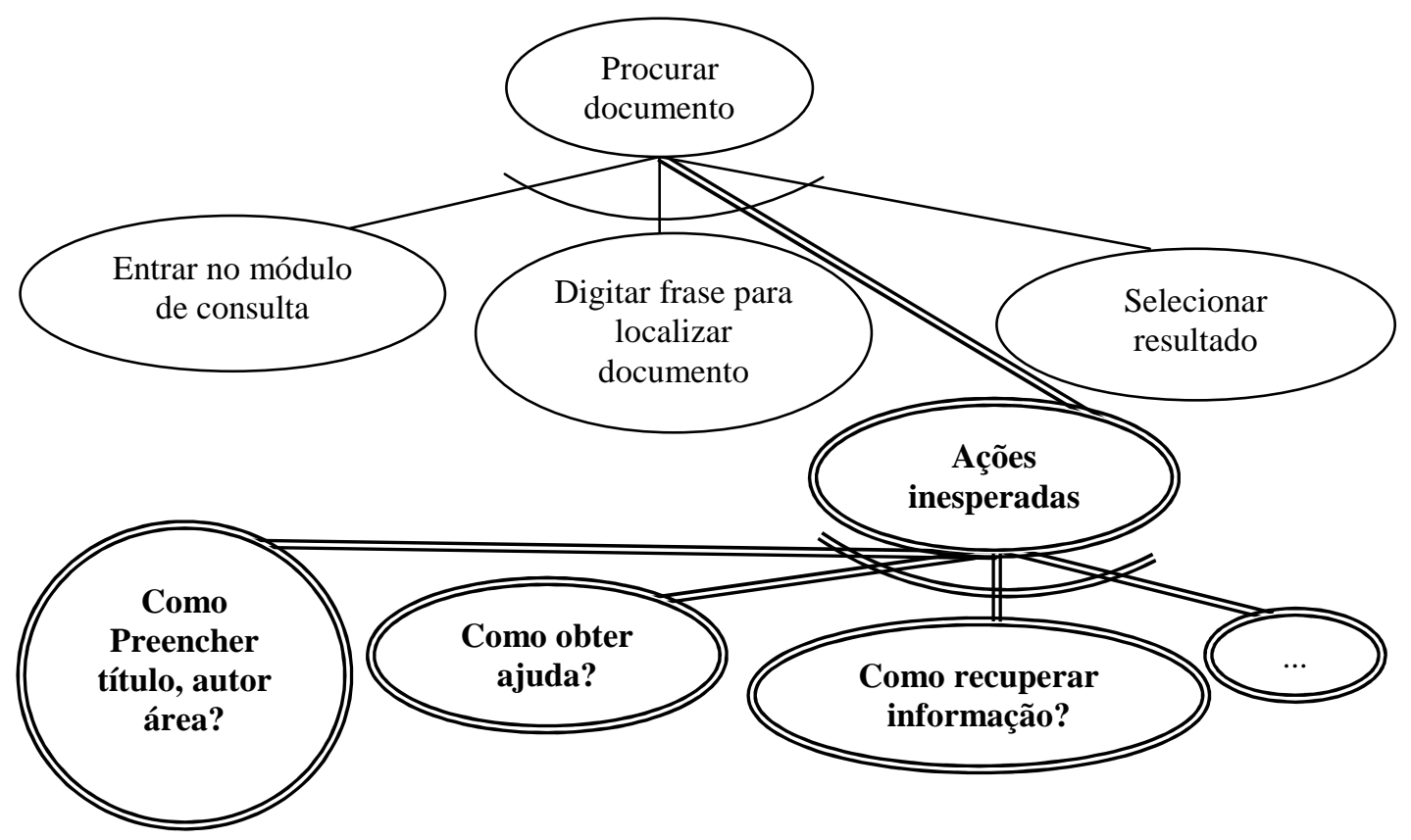


Figura 2 - Análise da atividade

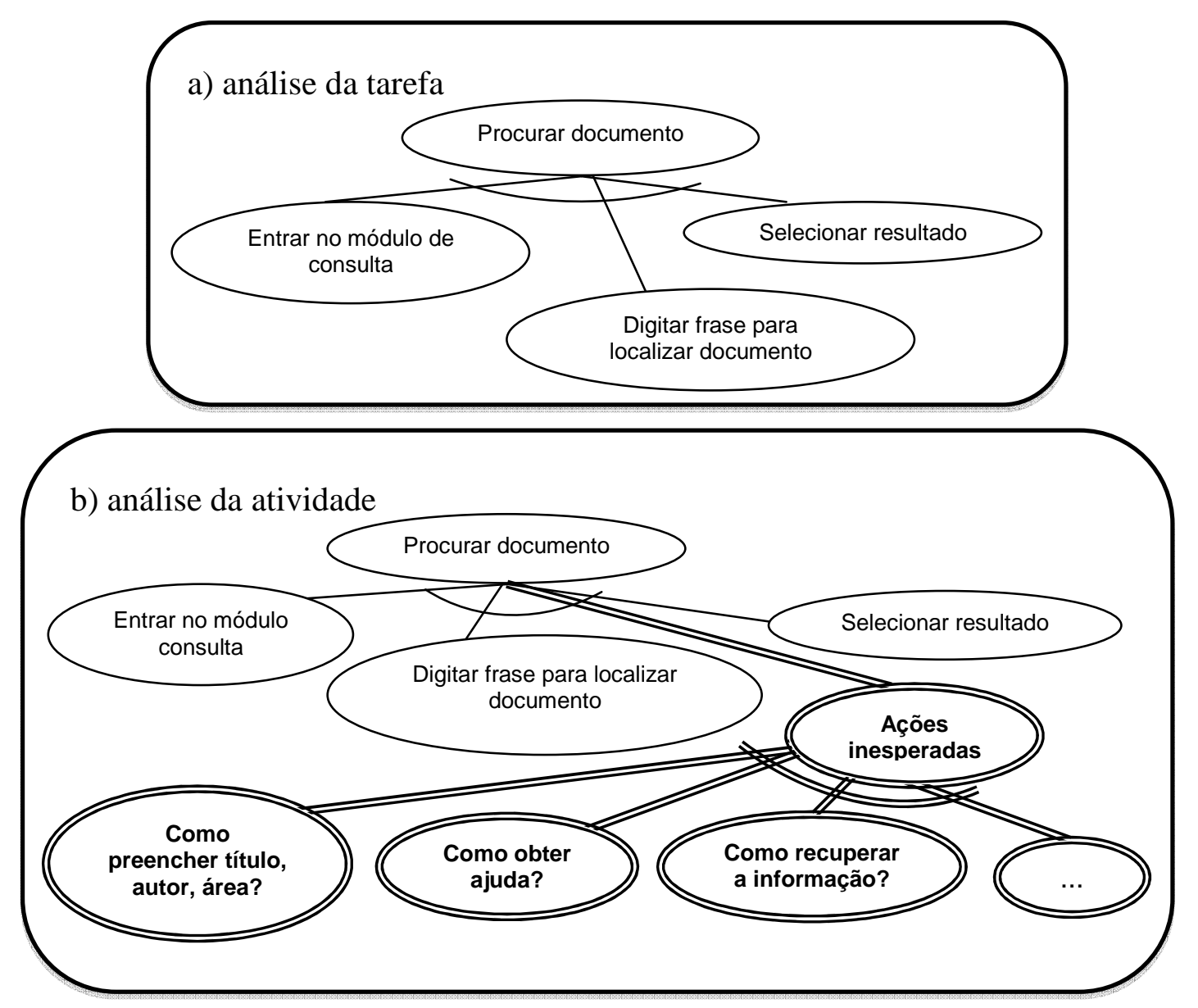


Figura 3 - Análise comparativa entre a tarefa (a) e a atividade (b)

\section{A gestão da informação centrada no usuário}

$\mathrm{Na}$ criação de recursos digitais, a personalização de serviços precisa levar em consideração o desenvolvimento cultural, social e contextual dos indivíduos (Tammaro; Salarelli, 2008). Na visão que F. W. Lancaster (apud King, 1985) tinha do futuro, ele apresentou muitas características desejadas num sistema de informação:

- transferir a dose precisa da informação, nem mais nem menos;

- apresentar a informação na linguagem dos usuários e num nível de compreensão apropriado para os diferentes tipos de usuários;

- proporcionar a informação no momento exato em que esta seja necessária (de acordo com o tipo de informação requerida);

- proporcionar informação na forma desejada: em papel, sem papel ou das duas maneiras;

- transferir a informação precisa sem demora, isto é, o tempo de resposta deve ser muito curto;

- entregar a informação no lugar que usuário deseje, seja no escritório, na residência ou em qualquer outro lugar;

- proporcionar a informação a baixo custo ou sem custo nenhum.

Parece que nenhum desses ideais chegou a ser alcançado na sua totalidade, mas os profissionais da informação, os analistas e os usuários, com o suporte da tecnologia da informação e telecomunicações, podem somar seus esforços e conhecimentos em direção a esses ideais.

Estudos realizados em diferentes períodos revelaram que a atividade pode ser influenciada pelo modelo mental que os usuários têm da tarefa. Por exemplo, usuários confiantes pensam que vão executar a tarefa com pouquíssimos passos e de curta duração, isto é, eles imaginam que a representação lacunar que têm da tarefa corresponde à realidade e ficam desapontados ao perceberem que são requeridos muitos outros elementos, inclusive que precisam vencer dificuldades e imprevistos (Kafure e Cunha, 2006). 
Deve-se poupar o tempo do usuário. De acordo com Grandjean (1998), as operações rápidas e precisas reduzem o risco de falhas, incidentes e erros na realização da tarefa. Mas, os usuários de interfaces para a gestão da informação têm manifestado dificuldades no momento da realização da tarefa (atividade), por exemplo, na criação de termos de busca da informação, quando não sabem da existência do vocabulário controlado. Se os modelos comportamentais de como as pessoas fazem perguntas tiverem sido considerados na elaboração de projetos de interfaces, então os projetos poderiam dar suporte às dificuldades que os usuários enfrentam, o que refletiria na aceitação do sistema de informação e, consequentemnte, no grau de satisfação dos usuários (Bates, 2003).

Havendo menos dificuldades a enfrentar, os usuários se concentrariam nos objetivos pretendidos, e, assim, alcançariam um melhor desempenho e otimização na busca da informação. A adaptação da interface às necessidades dos usuários tem resultado na visualização de opções simples em buscas avançadas e complexas. Disponibilizar a redefinição da frase de busca utilizando as opções avançadas da interface é um refinamento importante, quando se busca informação em bancos de dados mais complexos.

\section{O design emocional na gestão da informação}

A concepção interdisciplinar da interface centrada nos usuários, neste sentido, converge com a definição de Löbach (2001) ao afirmar que o desenho é o processo de adaptação do entorno objetual às necessidades físicas e psíquicas dos indivíduos. Em relação ao design emocional, este trabalho aborda um enfoque ligado ao conceito de usabilidade.

Para se ter sucesso na tomada de decisões, afirmava-se, tradicionalmente, que era necessário evitar a intervenção das emoções. Era frequente dissociar a emoção da razão em termos mentais e neurológicos. Hoje, estudos científicos ilustram como a emoção e a cognição são absolutamente entrelaçadas, e, também, esclarecem que a emoção auxilia na escolha entre diversas opções e possibilidades em complemento com o conhecimento e a razão (Damásio, 1994, 2005; Norman, 2004). De acordo com Cañada (2005), está demonstrado que a percepção sobre um objeto muda dependendo se ele é fácil de usar ou não, pois, segundo esse autor, nunca desenvolvemos vínculos emocionais positivos com algo cujo uso seja trabalhoso. Desta maneira, a relação entre emoção e usabilidade é direta.

Janczura (1999), em pesquisas de psicologia cognitiva, esclarece que os usuários da 
informação possuem diferenças que merecem ser estudadas com a finalidade de criar recursos eletrônicos e mecanismos para acessar a informação mais adaptados ao estilo de cada um, concluindo que:

- diferentes indivíduos procuram e processam a informação utilizando diferentes estratégias;

- diferentes estratégias podem ser mais ou menos efetivas para diferentes pessoas, em diferentes contextos;

- os indivíduos, em alguma medida, podem adotar consistentemente uma ou outra estratégia de processamento da informação (tais tendências são chamadas de estilos).

Diante da discrepância entre o modelo mental e a interface de sistemas de gestão da informação, nesta pesquisa aplica-se uma estratégia de regulação ${ }^{1}$ para adaptar a interface ao modelo mental dos usuários. É nesse momento que se obtêm e aplicam recomendações para recriar ambientes mais afetivos, tendo como meta afinar a usabilidade e o design emocional (Figura 4).

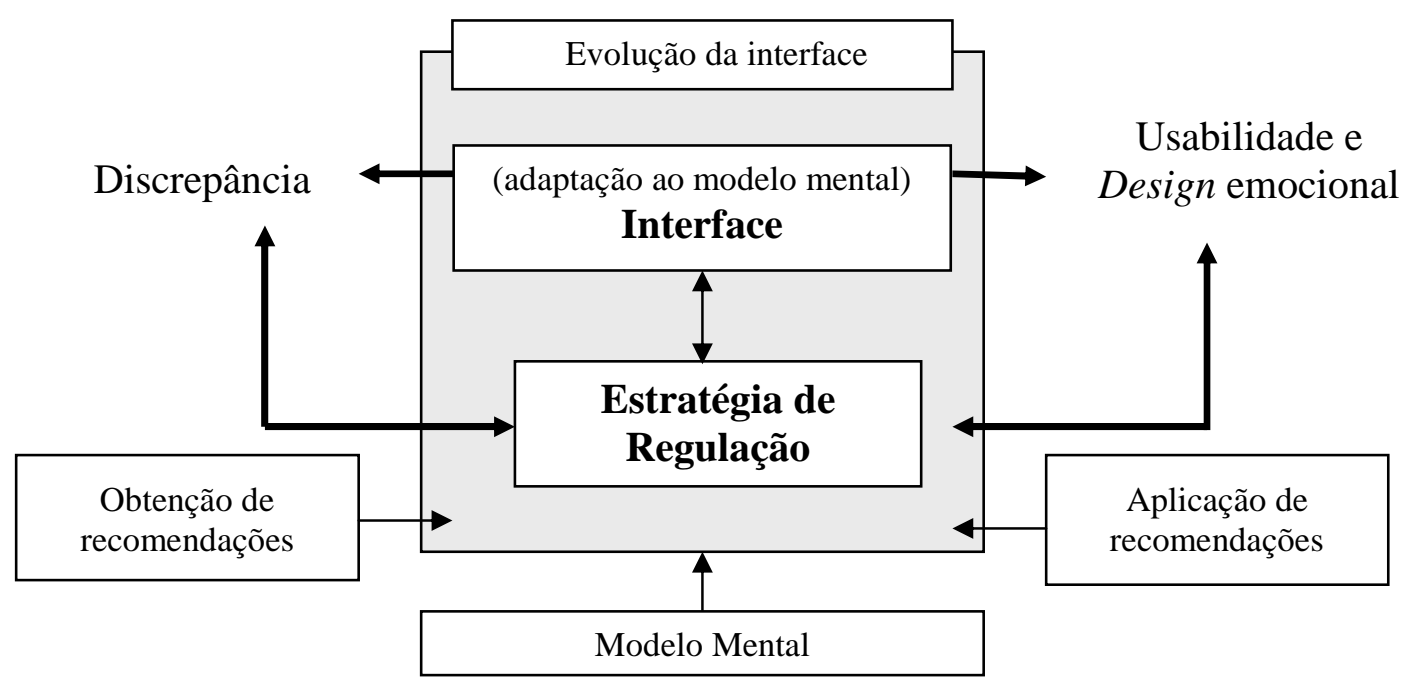

Figura 4 - Estratégia de regulação na adaptação da interface ao modelo mental Fonte: Adaptado de Kafure, 2004, p. 235.

\footnotetext{
${ }^{1}$ A estratégia de regulação, de acordo com Ferreira (2002), é utilizada na busca de soluções de problemas existentes em situações reais de trabalho. 
Ao se aplicar a avaliação da usabilidade no início do desenvolvimento de interfaces ou prototipagens em papel - nas quais têm participado usuários, profissionais da informação e analistas -, foi identificada a existência de uma discrepância inicial entre o modelo mental dos usuários, o dos profissionais da informação e o dos analistas. A discrepância diminui ao ser estabelecido um diálogo entre esses indivíduos, num processo de regulação interativo, por meio de técnicas como a observação, os grupos focais, as entrevistas, os arranjo de cartões e a análise da tarefa e atividade. O processo de regulação tem sido decomposto em criações parciais, interpondo juízos, atos de aceitação ou rejeição, para enriquecer o processo criativo da interface (Kafure et al., 2007, Kafure, 2009a).

\section{Aprendizagem e avanços obtidos}

Diversas experiências, tais como:

- catálogos públicos de acesso em linha (Kafure, 2004);

- biblioteca digital PREDICA (Plataforma experimental para sistemas de REcomendação, Descobrimento de conhecimento, desenvolvimento de Interfaces adaptativas e Consultas Avançadas), desenvolvida na Escola de Engenharia de Sistemas e Computação da Universidade del Valle, na Colômbia, financiada pelo Instituto Colombiano para o desenvolvimento da Ciência e da Tecnologia (Colciencias) e pela Universidade del Valle (Kafure et al., 2007);

- projetos de interfaces desenvolvidos nas disciplinas Usabilidade em Interfaces Humano-Computador na Escola de Engenharia de Sistemas e Computação da Universidade del Valle, em Cali, na Colômbia; Seminário 1: Usabilidade em Interfaces Humano-Computador; Seminário 2: Ergonomia Cognitiva no Departamento de Ciência da Informação e Documentação na Universidade de Brasília, no Brasil; Biblioteca Digital na Universidade Federal do Pará, Brasil; Usabilidade na Interação HumanoComputador e o Seminário em Arquivística 2: imagem \& cognição (Kafure, 2009a);

- Orientação em monografias de graduação em biblioteconomia e Ciência da Informação (Alves, 2007; Sousa, 2008; Fraga, 2008; Silva, 2009; Yunoki, 2009; Farias, 2010); 
- Orientação de Planos de Atividade Complementar (PACs): Aumento da usabilidade de ferramentas de avaliação de interfaces humano-computador; Identificação do perfil do usuário da informação na Arquivologia com a finalidade de promover a integração dos usuários e os conceitos arquivísticos desde o início, na concepção das interfaces de Sistemas Informatizados de Gestão Arquivística de Documentos (SIGADs); Usabilidade e Design Emocional na Gestão Arquivística de Documentos Escolares, projeto social em que são utilizadas as novas tecnologias, enfatizando a importância do desenvolvimento de um sistema de informação que facilite a comunicação entre os alunos, a escola e a família. É valorizada a participação da família na escola, pois a família pode propiciar os aportes afetivos necessários para melhorar a aprendizagem (Kafure, 2009b).

As pesquisas feitas têm permitido indagar, aprender, divulgar, verificar, demonstrar a existência de uma discrepância entre o modelo mental dos usuários (Figura 1) e a atividade (Figura 2), contribuindo também para suprir esta lacuna por meio da inclusão da objetividade da tarefa, da subjetividade e do design emocional dos usuários na geração e aplicação de recomendações para que a interface seja um suporte para:

- o aceso à informação e sua localização no ambiente de trabalho;

- a compreensão de elementos visuais e verbais;

- a facilidade e satisfação de uso, por meio de elementos visuais e verbais dentro de cada tela do ambiente de trabalho.

Isto tem agregado benefícios à interação do usuário com as interfaces dos sistemas para a gestão da informação, aumentado a usabilidade na recuperação da informação, inclusive antes de as interfaces serem utilizadas formalmente. Nas avaliações comparativas, os usuários expressa aceitação e satisfação em relação à adaptação das interfaces.

Em várias oportunidades tem sido ilustrado que, após a avaliação da usabilidade quando o design emocional e a eleição de elementos visuais e verbais passaram por adequações -, os usuários ficam mais satisfeitos com os benefícios decorrentes da interação humano-computador. 
Os avanços na pesquisa impulsionaram a criação do projeto e do Grupo de Pesquisa Informação, Design e Usabilidade, voltado para estudos interdisciplinares sobre usuários e interfaces de gestão da informação, abrangendo não só a parte acadêmica, mas também a responsabilidade social da Ciência da Informação. O PAC Usabilidade e Design Emocional na Gestão Arquivística de Documentos Escolares é um exemplo de estudo de caso sobre essa aplicação na Associação Pró-Educação Vivendo e Aprendendo, que resultou num contato mais específico com a realidade vivenciada na instituição em relação às famílias, visando acompanhar mais de perto o estímulo ao crescimento das crianças (Kafure, 2009b).

\section{Considerações finais}

A avaliação da usabilidade de sistemas para a gestão da informação se perfila como uma área de pesquisa específica no estudo de modelos de interfaces e comportamentos esperados pelos usuários. Se, com o acompanhamento dos usuários, desde as primeiras etapas da concepção das interfaces de gestão da informação, for levado em consideração o estabelecimento de modelos e comportamentos não desejados, eles serão úteis na avaliação da usabilidade do sistema.

A incorporação das novas tecnologias no desenvolvimento de sistemas para a gestão da informação propõe desafios inesperados para o estudo dos seus usuários, especialmente considerando as necessidades e expectativas dos seres humanos na interação com as interfaces dos computadores e na avaliação da usabilidade e design emocional.

O conceito de sistemas para a gestão da informação aparenta ser algo revolucionário, mas, na verdade, é o resultado de um processo gradual e evolutivo. As boas interfaces de sistemas para a gestão da informação necessitam de ajustes para se adaptarem, constantemente, aos seus usuários.

Considerar o usuário como uma "caixa preta", sem estudar as funcionalidades internas e emocionais de sua mente, examinar só as manifestações externas e visíveis de sua entidade mental, focar um projeto de interfaces orientado na psicologia do analista ou do profissional da informação, deixando de lado a cognição e o design emocional do usuário, seria negar a responsabilidade social da Ciência da Informação.

O aspecto comunicacional da interface humano-computador para a gestão da informação requer a inter-relação entre Ciência da Informação, Tecnologia da Informação e 
Comunicação, Artes Visuais, Ergonomia Cognitiva, Educação e áreas afins, pois cada área propõe métodos, técnicas e aspectos específicos para aumentar a usabilidade das interfaces e seu design emocional em relação aos usuários, à tarefa e à atividade.

Um modelo da tarefa estabelecido em colaboração com a análise da atividade pode levar a um modelo da interação humano-computador com alto grau de usabilidade, e, portanto, a usuários satisfeitos, com redução nos custos e no tempo de capacitação.

Se a informação existe para servir aos usuários, é primordial aumentar a usabilidade e o design emocional, num processo contínuo, para permitir que os usuários possam gerir a informação de maneira eficaz, eficiente e agradável.

\section{Referências bibliográficas}

ALVES, F. K. T. Avaliação da usabilidade do serviço de renovação em linha da Biblioteca Central da Universidade de Brasília. 2007. Monografia (Bacharelado em Biblioteconomia) Universidade de Brasília (UnB), 2007

ARNHEIM, Rudolf. Intuição e intelecto na arte. São Paulo: Martins Fontes, 1989.

BATES, M. J. Improving user access to library catalog and portal information. In: BICENTENNIAL CONFERENCE ON BIBLIOGRAPHIC CONTROL FOR THE NEW MILLENNIUM. Washington. 2003. Disponível em: http://www.loc.gov/catdir/bibcontrol/ bates.html. Acesso em: 2 jul. 2006.

CAÑADA, J. Entrevista sobre los paradigmas del diseño. In: Diseño emocional: definición, metodología y aplicaciones. Santiago de Chile, 2005.

CARD, S.; MORAN, T.; NEWELL, A. The Psychology of Human-Computer Interaction. Hillsdale, NJ: Lawrence Erlbaum Associates, 1983.

CUNHA, M. B. da. Notas de aula da disciplina: Fontes para a Recuperação da Informação. Brasilia: UnB/CID, 2003.

GREEN, M. W. The Design of User Graphical Interfaces. 1995. Ph. D. Thesis. Tech. Report CSRI-70, Computer Systems Research Institute, University of Toronto, Canada, April 1995.

DAMÁSIO, A. R. Descartes' error: emotion, reason, and the human brain. New York: Grosset, Putnam, 1994.

2005.

. Somos esclavos de las emociones y del entorno. El País [periódico], España, 21 oct. 
FARIAS, R. M. T. Usabilidade infantil: um olhar atento aos nativos digitais. Monografia de Graduação do Bacharelado em Biblioteconomia, UnB/FCl, 2010.

FERREIRA, M. C. O sujeito forja o ambiente, o ambiente "forja" o sujeito: a inter-relação indivíduo-ambiente em ergonomia da atividade. Brasília: Laboratório de Ergonomia, Instituto de Psicologia da UnB, 2002. Disponível em: <http://www.unb.br/ip/labergo/sitenovo/mariocesar/artigos3/OSujeito.PDF>.

FRAGA, C. L. Usabilidade do Portal de serviços e Informações do Governo Segundo o Ponto de Vista do Ciudadão. Monografia de Graduação do Bacherelado em Biblioteconomia, UnB/FCl, 2009.

GRANDJEAN, E. Manual de ergonomia: adaptando o trabalho ao homem. Porto Alegre: Bookman, 1998.

GUÉRIN, F. et al. Compreender o trabalho para transformá-lo: a prática da ergnomia. São Paulo: Edgar Blücher, 2001.

JANCZURA, G. A. Acessibilidade conceitual em tarefas de categorização e memória. Brasília: Universidade de Brasília, CNPq, 1999.

JOHNSON, P.; JOHNSON, H.; WADDINGTON, R.; SHOULS, A. Task-related knowledge strutures: analysis, modelling and application. In: JONES, M. D.; WINDER, R. (Eds.). People and computers IV. Manchester, UK: Cambridge University Press, 1988. p. 35-62.

JOHNSON, H.; JOHNSON, P. Task knowledge strutures: psychological basis and integration into system design. Acta Psychologica, n. 78, p 3-26, 1991.

KAFURE, Ivette. Validação do formalismo TAOS para a análise da tarefa no contexto da concepção de interfaces homem-computador. 2000. Dissertação (Mestrado) - Universidade Federal da Paraíba (UFPB), Campina Grande, 2000.

KAFURE, I. Usabilidade da imagem na recuperação da informação no catálogo público de acesso em linha. Tese (Doutorado) - Universidade de Brasília (UnB), Brasília, 2004. Disponível em: http://bdtd.bce.unb.br/tedesimplificado/tde_busca/arquivo.php?codArquivo = 1011 .

KAFURE, I.; CUNHA, M. B. Usabilidade de ferramentas tecnológicas para acesso à informação. Revista ACB, Florianópolis, v. 11, n. 2, p. 273-282, 2006.

KAFURE, I.; VALENCIA, M. E.; RODRIGUEZ, P. J.; FLORIÁN, B.; CARRILLO, J. E.; SOLARTE, O.; CIPRIAN, M. Evaluación de la Usabilidad de la Biblioteca Digital PREDICA. In: SEMINÁRIO INTERNACIONAL DE BIBLIOTECAS DIGITAIS, 2007, Brasil.

KAFURE, I. Usabilidad y diseño emocional en la gestión de la información. In: CONGRESSO INTERNACIONAL DE INTERAÇÃO PESSOA-ORDENADOR (Interacción 2009), 10. 2009, Armenia (Colombia). [Anais]. 2009a. 
KAFURE, I. Plano de Atividade Complementar: Usabilidade e design emocional na gestão arquivística de documentos escolares. Brasília: UnB, Departamento de Ciência da Informação e Documentação, 2009b.

KING, D. W. Obstáculos para futuros sistemas ideales de transferencia de información. In: TELECOMUNICACIONES y bibliotecas: um manual para bibliotecarios y directores de bibliotecas. México: Universidad Nacional Autónoma de México, 1985.

MARKOPOULOS, P.; PYCOCK, J.; WILSON, S.; JOHNSON, P. Adept: a task based design environment. In: Hawaii International Conference on System Sciences. 25 ${ }^{\text {th }}$, Mauai, 1992. Proceedings... IEEE Computer Society Press. 1992. Disponível em: $<$ http://www.idemployee.id.tue.nl/p.markopoulos/downloadablePapers/Markopoulos-P1992.HICSS25.pdf>.

MARKOPOULOS, P.; GIKAS, S. Formal specification of a task model and implications for interface design. Cognitive Systems, v. 4, n. 3-4, p. 289-310, Feb. 1997.

MORAN, T. The Command Language Grammar: a representation for the user interface of interactive computer systems. International Journal of Man-Machine Studies, v. 15, p. 3-50, 1981.

NORMAN, D. A. Some observations on mental models. In: GENTNER, Dedre; STEVENS, Albert L. Mental Models. Hillsdale, NJ: Lawrence Erlbaum Associates, 1983. p. 7-14.

NORMAN, D. A. Cognitive engineering. In: NORMAN, D. A.; DRAPER, S. W. (Eds.). User centered system design: new perspectives on human-computer interaction. Hillsdale, NJ: Erlbaum Associates, $1986.526 \mathrm{p}$.

NORMAN, D. A. Emotional design: why we love (or hate) everyday things. New York: Basic Books, 2004.

LÖBACH, B. Design industrial: bases para a configuração dos produtos industriais. São Paulo: Edgar Blücher, 2001.

MILLER, K. O Óleo de Lorenzo. Direção George Miller. Produção: Arnald Burk. Roteiro: Doug Mitchelle e George Miller. City studios: Universal pictures. Filme (1:34 min), 1992.

RICHARD, J. F. Les activités mentales: comprendre, raisonner, trouver des solutions. Paris: Armand Colin, 1990.

SACERDOTI, E. D. Planning in a hierarchy of abstraction spaces. Menlo Park, California, USA: Artificial Intelligence Center, Stanford Research Institute, 1974.

SCAPIN, D. L. Guide ergonomique de conception des interfaces homme-machine. France: INRIA, 1987. (Rapport INRIA n. 77). 
SCAPIN, D. L.; PIERRET-GOLBREICH, C. Towards a method for task description: MAD. France: INRIA, 1989.

SEBILLOTE, S. Théories et méthodolodies, décrire des taches selon les objetives des opérateurs de l'interview a la formalisation. Projet de Psychologie Ergonomique pour I'Informatique. France: INRIA Ronquencourt, 1991.

Task TD-5: methodology guide to task analysis with the goal of extracting relevant characteristics for interfaces, Esprit 3 Projet: P6593, "INTUITIVE". France: INRIA Ronquencourt, 1995.

SILVA, F. M. B. da. O impacto do design emocional na recuperação da informação no catálogo público de acesso em linha. Monografia de Graduação do Bacharelado em Biblioteconomia, UnB/CID, 2009.

SHNEIDERMAN, B. Designing the user interface: strategies for effective humman-computer interaction. $3^{\text {rd }}$ ed. Reading, MA: Addinson Wesley, 1998.

SOUSA, L. A. G. de. Avaliação da usabilidade em sítios na internet: um estudo de caso do site de Legislação da Presidência da República. 2008. Monografia (Bacharelado em Biblioteconomia) - Universidade de Brasília (UnB), 2008.

TAMMARO, A. M.; SALARELLI, A. A biblioteca digital. Brasília: Briquet de Lemos, 2008.

YUNOKI, B. T. Utilização do Moodle como ambiente de apoio ao ensino presencial: estudo de caso no curso de Biblioteconomia da Universidade de Brasília. Monografia de Graduação do Bacherelado em Biblioteconomia, UnB/CID, 2009.

\footnotetext{
i Professora Faculdade de Ciência da Informação/UnB. E-mail: ivettek@unb.br
} 